\title{
FRATELLI TUTTI: UNA INVITACIÓN A LA FRATERNIDAD DESDE EL HORIZONTE DE LA DIGNIDAD DE LA PERSONA ${ }^{I}$
}

\author{
FRATELLI TUTTI: AN INVITATION TO FRATERNITY \\ FROM THE HORIZON OF THE DIGNITY OF THE PERSON
}

\author{
SANDRO PAREDES-DÍAZ* \\ Mg. en Ciencias Religiosas y Filosóficas \\ sandroparedes@gmail.com \\ Universidad Católica del Maule, Chile
}

Cómo citar este artículo:

PAREDES, S. "Fratelli Tutti: Una invitación a la fraternidad desde el horizonte de la dignidad de la persona” en Palabra y Razón. Revista de Filosofía, Teología y Ciencias de la Religión. No 20 DICIEMBRE 202I, pp. I42-I52 https://doi.org/I0.29035/pyr.20.I42

I El siguiente texto corresponde a la participación en el "Coloquio en torno a la Encíclica Fratelli Tutti”, organizado por la Pastoral Universitaria de la Universidad Católica del Maule, el o3 de diciembre de 2020 


\title{
RESUMEN
}

Esta contribución breve propone un acercamiento a la encíclica Fratelli Tutti del Papa Francisco a partir del concepto clave de dignidad de la persona. Para esto se comentan y articulan las categorías de prójimo, cultura del encuentro y la amistad con los pobres, presentes en el documento. Estas son un modo de anunciar, en el mundo de hoy, la verdad trascendente de la dignidad de la persona.

Palabras claves: Papa Francisco / Fratelli Tutti / evangelización / dignidad de la persona / amistad social

\begin{abstract}
This brief contribution proposes an approach to the encyclical Fratelli Tutti of Pope Francis based on the key concept of dignity of the person. For this, the categories of neighbor, culture of encounter and friendship with the poor, present in the document, are discussed and articulated. These are a way of announcing, in today's world, the transcendent truth of the dignity of the person.
\end{abstract}

Keywords: Pope Francis / Fratelli Tutti / evangelization / dignity of the person / social friendship 


\section{Introducción}

A modo de introducción quisiera destacar que la encíclica Fratelli Tutti del Papa Francisco (3 de octubre de 2020) arriba en un momento preciso, el momento en que necesitamos como Iglesia y como sociedad recuperar la esperanza. La pandemia que estamos padeciendo ha venido a agudizar muchas situaciones y problemas que el Papa Francisco ha denunciado en instancias anteriores, como el individualismo, la cultura del descarte, entre otros ${ }^{2}$. En efecto, la Pandemia de Covid-ig la sufrimos en dos niveles. Por una parte, se trata de una amenaza global, que revela la fragilidad de los vínculos internacionales para enfrentar solidariamente el coronavirus. Por otra parte, sufrimos la pandemia en un nivel íntimo o privado. La pérdida de vidas humanas debido a la enfermedad, el sacrificio de tantos que luchan contra ella, el aislamiento y encierro que hemos tenido que vivir, la pérdida de fuentes de trabajo, la incertidumbre laboral, entre muchas otras. Como pocas veces hemos podido vivenciar la globalización en la interconexión de sus aristas mundiales y locales, lamentablemente desde esta dolorosa arista.

Desde esta perspectiva, la encíclica Fratelli Tutti intenta responder a esta crisis con un mensaje de esperanza sobre la fraternidad y la amistad social en estos dos niveles que hemos declarado: por una parte, es un llamado a soñar y retomar un proyecto común, una fraternidad universal, pero, por otro lado, es también un llamado a la conversión personal, a la forma en que hasta ahora hemos convivido para instalar una verdadera amistad social. Lo personal y lo comunitario, individuo y sociedad, está entrelazado permanentemente en esta encíclica, debido a lo cual no podemos pensar que solo una respuesta estructural puede hacernos alcanzar la anhelada fraternidad, como tampoco la sola respuesta individual puede dejarnos descansar. Se trata de una articulación que expresa la misma constitución de la identidad humana.

Estos dos niveles se articulan por medio del método de discernimiento que ha asumido la ética cristiana, que conocemos por sus momentos de Ver, Juzgar y Actuar. Gracias a este método, Fratelli Tutti hunde sus raíces en la realidad, en la historia de las personas

2 Por ejemplo, los conceptos de individualismo (EG 63, 67,89, 99; LS II9, 208) y cultura del descarte (EG 53; AL I9I; GE IOI; CV 78; LS I6, 20, 22) atraviesan de modo recurrente el magisterio del Papa Francisco. 
y de los pueblos, en sus problemas y sus angustias. Pero también se abre a la palabra de Dios y, desde allí, habla en un lenguaje común, un lenguaje irrenunciable que es la dignidad de la persona humana y nos invita a dialogar en una cultura del encuentro, a reconocernos en la humanidad compartida que en estos tiempos experimentamos desde su vulnerabilidad.

Con estas palabras quiero proponer una lectura pastoral y social de la encíclica del Papa Francisco, que nos permita recoger algunos llamados importantes para nuestras comunidades de modo que, como Iglesia, seamos verdaderos promotores de la fraternidad y amistad social a la que el Papa nos convoca.

\section{Hay un modo de anunciar la verdad}

En primer lugar, la Encíclica nos ofrece una clara actualización del evangelio, la cual tiene un matiz especial y muy necesario para alcanzar la fraternidad universal, pues se trata de una actualización de la forma del evangelio. En efecto, en la segunda parte de la Encíclica, el Papa Francisco se ha inspirado en el texto del Buen Samaritano (Lc IO, 25-37), donde reconoce que, si bien esta carta está dirigida a todas las personas de buena voluntad, más allá de sus convicciones religiosas, la parábola se expresa de tal manera que cualquiera de nosotros puede dejarse interpelar por ella.

A poco andar, el Papa nos hace entender que una clave de lectura de este pasaje bíblico es la categoría de prójimo. En efecto, si miramos con atención, en el texto hay dos maneras de entender este término. Para el doctor de la ley, el prójimo es comprendido como otro judío, alguien que forma parte de su nación y de su raza. Para Jesús, en cambio, la categoría de prójimo es universal. Se trata más bien de hacerse prójimo del que está a la orilla del camino. Si hasta el enemigo del judío en ese entonces, el samaritano, se ha hecho prójimo del hombre caído actuando con misericordia. En este sentido, el samaritano vive en su corazón la misericordia que Dios tiene para todos: "La misericordia de cada persona se extiende a su prójimo, pero la misericordia del Señor alcanza a todos los vivientes" (Sirácida I8,13).

Por otra parte, el Papa nos recuerda el vínculo de este amor fraterno con el mismo misterio de Dios, a tal punto que la negación del hermano es una negación de Dios mismo. La primera Carta de Juan lo afirma claramente: "Quien ama a su hermano permanece en la luz y no tropieza. 
Pero quien aborrece a su hermano está y camina en las tinieblas" (I Jn 2,IOII). No descuida el texto el carácter soteriológico de este amor fraterno: "Nosotros sabemos que hemos pasado de la muerte a la vida, porque amamos a los hermanos. Quien no ama permanece en la muerte" (I Jn 3,I4). E incluso una dimensión gnoseológica, como camino para conocer a Dios: "Quien no ama a su hermano, a quien ve, no puede amar a Dios, a quien no ve" (I Jn $4,20)$.

Lo que el Santo Padre nos propone es una actualización de la verdad más profunda del evangelio, que se traduce en lo que denominamos "dignidad de la persona humana". El amor de Dios se transcribe como dignidad de toda persona. Esta actualización le permite al Papa Francisco proponer el respeto a la dignidad de la persona humana como el principio trascendente que necesita la comunidad internacional para alcanzar una verdadera fraternidad.

Lo que deseo destacar con esto es que el Papa nos ofrece una lectura actualizada de la perenne verdad del evangelio, en la cual nos podemos reconocer no solo como miembros de la Iglesia, sino como seres humanos, miembros de una familia universal. Ahora, como indicábamos más arriba, nos parece que el Papa no solo propone una actualización de la verdad del evangelio, sino también un camino, una forma o modo de anunciarlo desde la identidad más profunda del cristiano.

Al inicio de la Encíclica, el Papa Francisco trae a colación el episodio de la vida de san Francisco de Asís cuando visita al Sultán Malik-elKamil, en Egipto. Nos ofrece allí una maravillosa descripción de la figura de san Francisco: "La fidelidad a su Señor era proporcional a su amor a los hermanos y a las hermanas". El texto nos dice que san Francisco fue al encuentro del Sultán con la misma actitud que pedía a sus discípulos: "que, sin negar su identidad, cuando fueran «entre sarracenos y otros infieles [...] no promuevan disputas ni controversias, sino que estén sometidos a toda humana criatura por Dios" (n. 3). El papa nos hace ver que esto, en aquella época, era un pedido extraordinario y que impresiona que san Francisco invitara a evitar toda forma de agresión o contienda. Seguidamente, en el n. 4 afirma: "Él no hacía la guerra dialéctica imponiendo doctrinas, sino que comunicaba el amor de Dios".

El papa Francisco nos exhorta al amor como el modo de anunciar el evangelio, que es el único que se condice con el mensaje que se quiere anunciar. El anuncio del amor exige coherentemente el camino del amor. El llamado hacia el respeto de la dignidad de la persona humana exige el camino de la fraternidad y amistad social, el camino de la amabilidad, 
como lo recuerda en el n. 222.

Creemos que, en este sentido, el Papa Francisco ofrece un criterio de discernimiento para todas las comunidades a la hora de anunciar el evangelio. No basta con preguntarse si lo que debo decir es buena noticia, verdad y evangelio. También hay que discernir la forma en que este mensaje es anunciado. Desde esta perspectiva, toda apología cristiana que no discierna sobre sus modos, no está capacitada aún para ser testigo del amor. Creo que esta dimensión de la encíclica nos coloca en el centro de la misión de Jesús, la cual no estuvo exenta de tentación respecto al modo de cómo anunciar el Reino 3 . El camino que asumió el Maestro no es el de la fama y reconocimiento, sino el del siervo sufriente. Creemos que estas reflexiones en torno al modo de anunciar la verdad sobre el ser humano y el camino de la fraternidad están en sintonía, pues no se construye una comunidad universal a partir de estructuras impuestas, sino desde la articulación de la libertad personal y el bien común.

\section{La cultura del encuentro}

En segundo lugar, quisiera destacar la propuesta de una cultura del encuentro que la Encíclica ofrece como uno de los modos de construir fraternidad. La cultura del encuentro es como una medicina, la vacuna tan anhelada contra el aislamiento y la soledad que este contexto de pandemia ha agudizado, porque ella es cercanía (Cfr. n.30). El término aparece reiteradas veces en el texto dándonos varios matices de los cuales deseo rescatar solo tres.

Detrás de esta categoría está la idea de apertura hacia el otro, apertura que hunde sus raíces en la misma antropología cristiana. Somos creados como seres en relación, "un ser social" como lo define el Concilio Vaticano II en Gaudium et Spes (n. I2). Por tanto, la apertura al otro, especialmente al extranjero, como menciona el Papa Francisco, es una de nuestras más propias posibilidades como seres humanos, la posibilidad que nos hace reencontrarnos con nuestra propia humanidad. Por eso entendemos las palabras que Francisco refiere al miedo en Fratelli Tutti, como uno de los enemigos de la cultura del encuentro: "El miedo nos priva así del deseo y de la capacidad de encuentro con el otro" (n. 4I).

3 En efecto, el relato sobre las tentaciones de Jesús en el desierto (Mt 4, I-II; Lc 4, I-I3) puede interpretarse desde esta perspectiva: las tentaciones que Satanás hace a Jesús apuntan al modo cómo realizar su misión, no a desistir de ella. Le propone un modo "glorioso" de presentarse a los hombres, de forma que todos lo admiren. El camino del siervo sufriente, por su parte, asume la vía de la humildad. 
Es necesario relacionar esta categoría de la cultura del encuentro con los conceptos de aprendizaje y de cambio. Podríamos decir que las dos últimas encíclicas del Papa Francisco son fruto de un ejercicio de encuentro con otros. La Encíclica Laudato Si puede interpretarse como un guiño a la cultura de oriente fruto del encuentro con el Patriarca Ecuménico Bartolomé. El Papa aprende en este encuentro, y esto provoca un cambio, una enriquecida mirada hacia nosotros mismos y nuestra casa común. En el caso de Fratelli Tutti, podemos reconocer el mismo gesto hacia la religión musulmana. Nuevamente, en una verdadera actitud de encuentro y apertura al otro, el Papa aprende, y esto provoca un cambio. En este caso, el Papa agradece el encuentro con el Gran Imán Ahmad Al-Tayyeb, quien le recordó que Dios "ha creado todos los seres humanos iguales en los derechos, en los deberes y en la dignidad, $y$ los ha llamado a convivir como hermanos entre ellos" (n. 5). Como podemos apreciar, siempre que hay una verdadera acogida del otro, hay un cambio positivo en la forma de mirarnos. El Papa Francisco, en este caso, nos da un hermoso ejemplo.

Francisco no entiende este encuentro con el otro como una forma de buscar una homogenización que destruye la identidad personal. La riqueza del encuentro es que cada uno, siendo lo que es, se abre al otro cuando hay un proyecto común de fraternidad. En este sentido, el encuentro tiene un fundamento en la verdad trascendente de la dignidad de la persona humana, verdad que brota del mismo evangelio. Solo desde esta llamada (vocación) podemos descubrir el profundo significado de la fraternidad, que desde este carácter trascendente se eleva por encima de los intereses individuales y de poderes fácticos.

El tercer aspecto de la cultura del encuentro que deseamos destacar es que ella es un camino o un rostro de la conversión. Fratelli Tutti evoca en varios momentos la encíclica del Papa emérito Benedicto XVI, Deus Caritas est. Allí nos decía: "No se comienza a ser cristiano por una decisión ética o una gran idea, sino por el encuentro con un acontecimiento, con una Persona, que da un nuevo horizonte a la vida y, con ello, una orientación decisiva" (DCE, I). El encuentro con la persona de Jesús no nos puede dejar indiferentes. Como dice el papa Benedicto, este encuentro otorga un nuevo horizonte a la vida, pues el núcleo de la conversión cristiana es el encuentro con una persona que nos transforma. La cultura del encuentro en Fratelli Tutti no pretende ser una discusión o un enfrentamiento de doctrinas, sino que es, en primer lugar, apertura y diálogo con el otro, un encuentro transformador entre personas. En este sentido, la cultura del encuentro es una extensión en el ámbito de la vida pública de la profunda experiencia 
de la conversión.

Si miramos a nuestras comunidades, creo que los distintos llamados a ser una "Iglesia en salida", encuentran en la cultura del encuentro una consolidación y una nueva posibilidad. Una iglesia en salida será, entonces, la Iglesia que es capaz de encontrarse con los otros miembros de la sociedad, capaz de dialogar con las juntas de vecinos, con las agrupaciones culturales y ecológicas, con los sindicatos, con otras comunidades, con otras religiones. Cuando se habla de la dignidad de la persona humana, todos podemos encontrarnos en la mesa del diálogo. La cultura del encuentro es un desafío para nuestras comunidades, porque también -no lo perdamos de vista- en ese encuentro con los hermanos se fragua nuestra permanente conversión.

\section{Construir fraternidad desde nuestra amistad con los pobres}

El tercer aspecto de la encíclica que deseamos destacar es la fidelidad a los pobres. En el número i8 el Papa aborda el tema de la cultura del descarte, donde critica el criterio de utilidad que sacrifica la vida de los más pobres, especialmente las personas mayores y con discapacidad. Pero son pobres también las mujeres vulneradas, y doblemente pobres, afirma el Papa, ya que "son las mujeres que sufren situaciones de exclusión, maltrato y violencia, porque frecuentemente se encuentran con menores posibilidades de defender sus derechos" (n. 23). Es interesante esta sección de la encíclica en que el Papa nos invita a mirar nuestra sociedad y a superar la cultura del descarte desde el criterio de los derechos fundamentales de la persona humana y darnos cuenta que el pobre es también quien es vulnerado en sus derechos. No podemos, entonces, mirar al pobre solo desde una categoría socioeconómica, sino que la recuperación de su dignidad pasa también por una dimensión jurídica coherente con su dignidad de persona. El punto de vista de la dignidad de la persona nos permite ampliar nuestra comprensión de lo que significa pobreza y de los ámbitos de nuestra acción pastoral.

Para el Papa Francisco, los pobres nos enseñan lo que es la verdadera solidaridad, que no puede entenderse como un "acto de generosidad esporádico" (n. II6). Francisco nos ofrece una definición de solidaridad que hunde sus raíces en la experiencia de los movimientos populares. Nos dice que solidaridad es:

"pensar y actuar en términos de comunidad, de prioridad de la vida de todos sobre la apropiación de los bienes por parte de 
algunos. También es luchar contra las causas estructurales de la pobreza, la desigualdad, la falta de trabajo, de tierra y de vivienda, la negación de los derechos sociales y laborales. Es enfrentar los destructores efectos del Imperio del dinero. [...] La solidaridad, entendida en su sentido más hondo, es un modo de hacer historia y eso es lo que hacen los movimientos populares" (n. II6).

La lucha de los pobres por su dignidad nos enseña la necesidad de volver a proponer la función social de la propiedad. Se trata, para el Papa Francisco, de hacerse cargo de nuestra cuota de responsabilidad respecto a su carencia. La encíclica trae a colación el testimonio de los Padres de la Iglesia: "Cuando damos a los pobres las cosas indispensables no les damos nuestras cosas, sino que les devolvemos lo que es suyo" (n. II9). Creemos que Fratelli Tutti nos ofrece una nueva mirada sobre la solidaridad, que solo se vislumbra desde la clave de la fraternidad y desde el lente de la dignidad de la persona humana. Dar es, entonces, devolver, como un acto de reconocimiento de lo que te corresponde por dignidad. En este sentido, la solidaridad es una expresión o un gesto de reparación de los derechos vulnerados.

En el capítulo V de la Encíclica sobre La mejor política, el papa Francisco afirma que la mejor ayuda para un pobre, el mejor camino hacia una existencia digna, es el trabajo (n. I62). Por ello es que insiste en que "ayudar a los pobres con dinero debe ser siempre una solución provisoria para resolver urgencias. El gran objetivo debería ser siempre permitirles una vida digna a través del trabajo" (n. I62). He aquí que la política encuentra una de sus tareas más nobles y urgentes, pues solo el trabajo digno puede permitir en una sociedad desarrollada no solo ganarse el pan, sino un cauce para el crecimiento personal, para establecer relaciones sanas, para expresarse a sí mismo, para compartir dones, para sentirse corresponsable en el perfeccionamiento del mundo y, en definitiva, para vivir como pueblo (Cfr. n. I62).

El Papa nos hace una invitación a establecer una amistad con los pobres pues solo la cercanía que nos hace amigos ha de permitirnos apreciar profundamente los valores que poseen, sus legítimos anhelos y su modo propio de vivir la fe. Por eso es que la Encíclica nos dice: " $\mathrm{La}$ opción por los pobres debe conducirnos a la amistad con los pobres" (n. 234). Es decir, que el proyecto de una fraternidad universal solo es posible si se transita el camino del encuentro auténtico con los pobres, encuentro que suscita la amistad con ellos y con todo el género humano. Nuevamente podemos percibir este llamado respecto al "modo" de anunciar la verdad 
evangélica. No se trata de una "política solidaria mancomunada" que construya una "estructura fraterna". Se trata del encuentro verdadero con el otro, encuentro que crea amistad. Muchas veces la mirada sobre el pobre es solo de "destinatario", pero pocas veces los vemos como amigos. Las soluciones profundas a sus dificultades y necesidades solo se alcanzan cuando se ha podido entablar una amistad sincera que logra sufrir y alegrarse con ellos. Se trata de salir más allá de los círculos de relaciones cercanas y hacernos cercanos nosotros a los pobres, en la clave de "hacerse prójimo".

Creo que estas y otras notas que podemos recoger sobre esta fidelidad a la opción preferencial por los pobres, nos puede ayudar a renovar el servicio que ciertamente realizan muchos grupos de voluntariado y comunidades. Lo que Fratelli Tutti nos ofrece con la reflexión sobre la amistad con los pobres es un criterio para toda acción social, sea esta comunitaria o personal. Pero creemos que también es un criterio para avanzar hacia una auténtica conversión, criterio que se puede formular en la pregunta: ¿Somos, como comunidad, amigos de los pobres? ¿Forman parte de mis amigos los pobres?

\section{Conclusión}

Para concluir, solamente quisiéramos destacar que la encíclica Fratelli Tutti nos hace un profundo llamado eclesial. Es verdad que ella ha sido escrita para convocar a todos los hombres y mujeres hacia la esperanza de la fraternidad y la amistad social, pero también es cierto que ella expresa al mismo tiempo el núcleo de nuestra fe. Ya en Lumen Gentium resonaba la fraternidad universal como naturaleza y misión de la Iglesia: "porque la Iglesia es en Cristo como un sacramento, o sea, signo e instrumento de la unión íntima con Dios y de la unidad de todo el género humano" (LG I). Es más, el diagnóstico que en aquella época realizaba el Concilio Vaticano II, apuntaba a la necesidad de un vínculo más profundo entre los seres humanos que los otorgados por una incipiente globalización: "Las condiciones de nuestra época hacen más urgente este deber de la Iglesia, a saber, el que todos los hombres, que hoy están más intimamente unidos por múltiples vínculos sociales, técnicos y culturales, consigan también la plena unidad en Cristo" (LG I). Este diagnóstico es compartido todavía por Fratelli Tutti, a pesar de haber cumplido más de cincuenta años.

Lo que nos pide el Papa Francisco con esta última encíclica es no abandonar este proyecto de fraternidad universal, que es el proyecto de Dios para la humanidad. En este sentido, este documento es una 
propuesta que nos renueva en nuestra misión como Iglesia y, desde esta mirada, Fratelli Tutti es un texto profundamente eclesial. El misterio de Cristo y el Misterio de la Iglesia salen al encuentro con el mundo de hoy en el lenguaje de la dignidad de cada persona. En la lucha por respetar y promover sus derechos todos nos debemos reconocer como hermanos.

Por último, en el contexto sobre la pregunta por la identidad cristiana en la sociedad actual, por lo que significa ser cristiano hoy, el texto del Papa Francisco nos propone una traducción concreta del núcleo del evangelio de Jesús en lo que considera la verdad trascendente de la dignidad de la persona. Esta verdad nos convoca como hermanos en la fe y miembros de la familia humana. Los cristianos no se extravían de la verdad del evangelio ni pierden su identidad cuando luchan por los derechos de los más desvalidos y el respeto por la dignidad de la persona. $\mathrm{Al}$ contrario, en el esfuerzo de un encuentro con el mundo actual, esta categoría se nos ofrece como camino para la acogida y construcción de una fraternidad universal. 